\title{
An Embedded System of Face Recognition Based on ARM and HMM
}

\author{
Yanbin Sun ${ }^{1,2}$, Lun Xie ${ }^{1}$, Zhiliang Wang ${ }^{1}$, and Yi $\mathrm{An}^{2}$ \\ ${ }^{1}$ Department of Electronic Information Engineering, School of Information Engineering, \\ University of Science and Technology, Beijing, 100083, China \\ ${ }^{2}$ School of Information, Hebei Polytechic University, Tangshan, Hebei 063009, China \\ v4lcasohu.com, \\ ygao@mail.tsinghua.edu.cn
}

\begin{abstract}
Face recognition is important for many applications, including security check-up, judicial administration, visual monitoring and intelligent interaction. Current commercial and research systems use software implementation and require a dedicated computer for the image-processing task--a large, expensive, and complicated-to-use solution. In order to make face recognition ubiquitous, the system complexity, size, and price must be substantially reduced. This paper presents an ARM-based embedded system for face recognition. The float image-pretreatment algorithm was redesigned to enable highly image-pretreatment implementation for ARM9 microprocessor. The system uses a CMOS digital imaging sensor OV7640, a S3C2410A processor and the Linux Operation System for the image processing. A face recognition algorithm based on the HMM is presented. The software can be run in intelligent mobile telephone or PDA directly. This new design is suitable for face detection and recognition, thus making an important step towards low-cost and portable systems.
\end{abstract}

Keywords: embedded system, Linux, HMM, ARM, Face Recognition.

\section{Introduction}

Compared with traditional human identification system, the embedded system of face recognition has many advantages. It's low-cost, simple-to-use, no dedicated image sensor. Face recognition method, which belongs to no-violation and initiatively method, does not interfere the person being identified and does not violate person's privacy, so it's easily accepted.

This paper describes the development of ARM9 embedded system of face recognition, based on the theory of HMM, and presents the design and practice of hardware and software. The system has the functions of image-capturing, facedetecting and face-recognizing. Moreover the float image-pretreatment algorithm is optimized, which increases the system processing speed.

The software of the system can be applied to the mobile phone with the Linux operation system. Thus face images can be captured through the built-in camera and data analyzed. Identification can be accomplished by comparing the result with the face information database. 


\section{System Frame and Design Scheme}

This system adopts the S3C2410A microprocessor with the ARM 920T RISC core produced by Samsung. The excellent process ability of S3C2410A microprocessor makes itself the first choice for developing portable devices [1]. To meet the demand of the video capturing of intelligent mobile phone, the system adopts the USB-busbased video model. The technics concerning the capturing, processing, storing, transmitting of images and HMM algorithm have been used in the system. The system frame is shown in Fig.1.

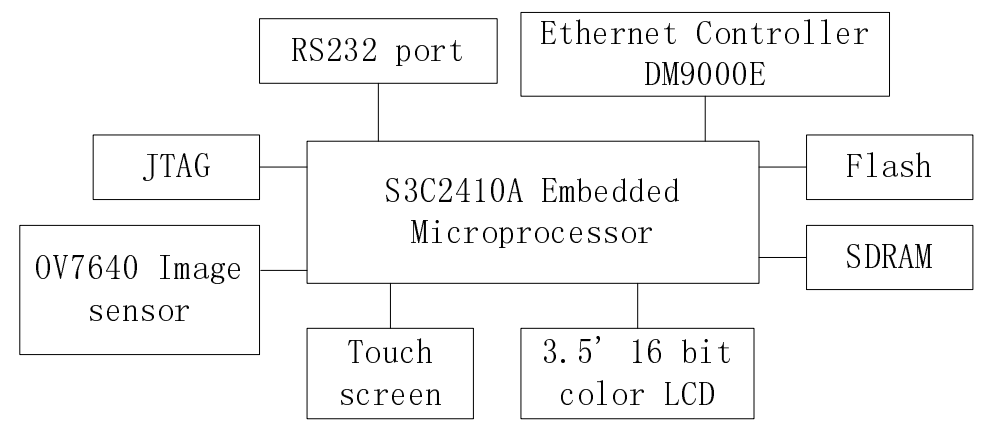

Fig. 1. The frame of ARM-based face recognition system

\section{Image-Preprocessing and Face Recognition Algorithm}

The first step of face recognition is to judge whether a face exists in the captured image or video data. If there exists, the following step is to work out the data about the situation and size of the facial organs. All the data makes a specific face, which is to compared with the face database. Through this, identity is recognized [2].

The process of face recognition falls into image preprocessing, face detecting, and face identifying.

\subsection{Image Preprocessing and Algorithm Optimizing}

Human being falls into the yellow, white, brown, and black in skin color. And this system focuses on the recognition of the yellow and white face.

The images from image sensor are presented in RGB method which is not the best model of skin color for computer recognition. Therefore the images are normally transformed from RGB to YUV to be processed. In YUV color model, brightness value can be easily separated and the calculation is simple, so this model is widely used $[3,4]$.

The formula of transforming RGB to YUV is as follows:

$$
\left[\begin{array}{l}
\mathrm{Y} \\
\mathrm{U} \\
\mathrm{V}
\end{array}\right]=\left[\begin{array}{ccc}
0.299 & 0.587 & 0.114 \\
-0.148 & -0.289 & 0.437 \\
0.615 & -0.515 & -0.100
\end{array}\right]\left[\begin{array}{l}
\mathrm{R} \\
\mathrm{G} \\
\mathrm{B}
\end{array}\right]
$$


For the lack of floating-point assist-processor of S3C2410A microprocessor, the calculating of the above formula is time-consuming. In order to increase the transforming speed, the floating-point formula is optimized into the formula of integer calculating and shift calculating.

$$
\left[\begin{array}{l}
\mathrm{Y} \\
\mathrm{U} \\
\mathrm{V}
\end{array}\right]=\left[\begin{array}{ccc}
38 & 74 & 15 \\
-19 & -37 & 61 \\
79 & -66 & 13
\end{array}\right]\left[\begin{array}{l}
\mathrm{R} \\
\mathrm{G} \\
\mathrm{B}
\end{array}\right] \gg>7
$$

The experiment proves that this formula greatly increases the transforming speed of the system from RGB to YUV.

The gray images and histograms transformed from the same captured image according to the above formulas are shown in Fig.2.
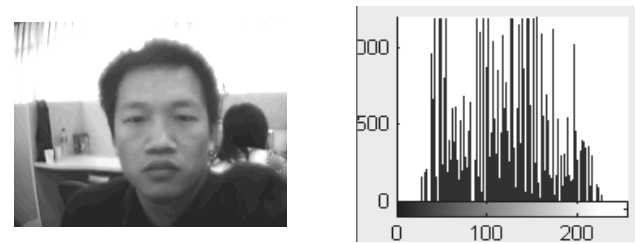

The gray images and histograms transformed from according to the float formula (1)
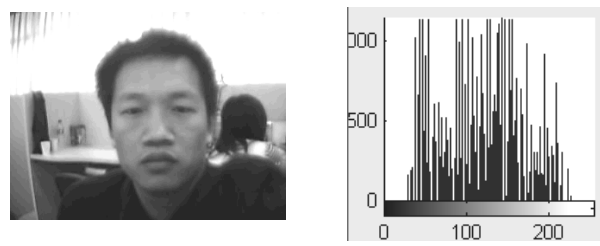

The gray images and histograms transformed from according to the integer formula (2)

Fig. 2. Emulating result

From the above data, it is seen that the minor difference between the transforming results of the two formulas has no effect on the following steps.

In the gray image, the value of one point decides whether it is skin location. By a threshold, the gray image can be transformed into two values image, in which 1 is skin location and 0 is not. From the two values image, the initial matrix is worked out by the boundary-based method. Thus a boundary rectangle is decided which covers

the skin area. The final output matrix is worked out through area-merging for the initial matrix[2].

\subsection{Face Detecting and Recognition Based on HMM}

Face detecting is to locate the feature areas in the output matrix and separate them. Face recognition is to compare the output feature face with the database. And then the corresponding label of the recognized face is outputted. They are closely related. Face detecting and recognition can be carried out on the base of HMM at the same time [5]. 
1. General concept of Hidden Markov Model (HMM)

HMM is a statistic model for feature signals. It consists of Markov chain of hidden, invisible and limited states and a probability density function. Markov chain includes probability distribution function of initial state and probability matrix of transferred state. Probability density function is related with those states.

HMM can be described using parameters as follow:

(1) Element $\mathrm{N}$ : it is the states number in the model. Supposing $\mathrm{S}$ is state space, and then $\mathrm{S}=\left\{\mathrm{S}_{1}, \mathrm{~S}_{2}, \hbar, \mathrm{S}_{\mathrm{N}}\right\}$. The model state at time $\mathrm{t}$ is $\mathrm{q}_{\mathrm{t}} \in \mathrm{S}, 1 \leq \mathrm{t} \leq \mathrm{T} . \mathrm{T}$ is the length of observing serial. The states serial of the model is $Q=\left\{\mathrm{q}_{1}, \mathrm{q}_{2}, \hbar, \mathrm{q}_{\mathrm{T}}\right\}$.

(2) Element $\mathrm{M}$ : it is the possible observed states number in each state. The observed states are marked as $\mathrm{V}=\left\{\mathrm{v}_{1}, \mathrm{v}_{2}, \hbar, \mathrm{v}_{\mathrm{m}}\right\}$ and each observed state is corresponding to one movement state.

(3) States transferring probability distribution $\mathrm{A}=\left\{\mathrm{a}_{\mathrm{ij}}\right\}$, in which $\mathrm{a}_{\mathrm{ij}}=\mathrm{P}\left(\mathrm{q}_{\mathrm{t}}=\mathrm{S}_{\mathrm{j}} \mid \mathrm{q}_{\mathrm{t}-1}=\mathrm{S}_{\mathrm{i}}\right), 1 \leq \mathrm{i}, \mathrm{j} \leq \mathrm{N}$. The condition is $0 \leq \mathrm{a}_{\mathrm{ij}} \leq 1$, and $\sum_{j=1}^{N} a_{i j}=1$

(4) The observed states probability distribution in state j: $B=\left\{b_{j}(k)\right\}$, $\mathrm{b}_{\mathrm{j}}(\mathrm{k})=\mathrm{P}\left(\mathrm{o}_{\mathrm{t}}=\mathrm{v}_{\mathrm{k}} \mid \mathrm{q}_{\mathrm{t}}=\mathrm{S}_{\mathrm{j}}\right), 1 \leq \mathrm{j} \leq \mathrm{N}, 1 \leq \mathrm{j} \leq \mathrm{M} . \mathrm{o}_{\mathrm{t}}$ is observed signal at time $\mathrm{t}$, and observed serail is $\mathrm{O}=\left\{\mathrm{o}_{1}, \mathrm{o}_{2}, \hbar, \mathrm{o}_{\mathrm{T}}\right\}$.

(5) Initial state distribution $\Pi=\left\{\pi_{\mathrm{i}}\right\}, \pi_{\mathrm{i}}=\mathrm{P}\left(\mathrm{q}_{\mathrm{i}}=\mathrm{S}_{\mathrm{i}}\right), 1 \leq \mathrm{i} \leq \mathrm{N}$.

HMM can be abbreviated as $\lambda=\{A, B, \Pi\}$. The input is limited character set $\mathrm{V}=\left\{\mathrm{v}_{1}, \mathrm{v}_{2}, \hbar, \mathrm{v}_{\mathrm{m}}\right\}$, so HMM is named as scattering HMM[6].

2. HMM for face recognition

According to the types of states transferring, HMM can be divided into ergodic and left-right. The transferring states of ergodic is random, and the state can transferred from itself to any other state. The state of the latter can only transfer from itself to the next.

The distribution of face feature is invariable from top to bottom and from left to right. So face can be modeled by 1D-HMM as is shown in Fig.3.

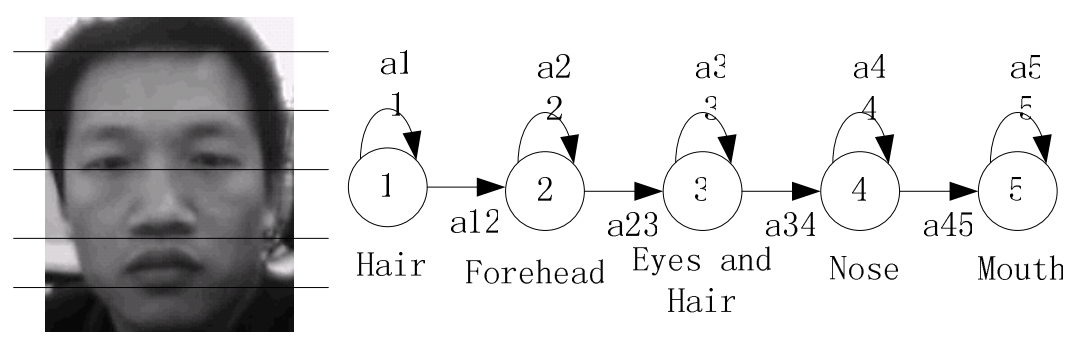

Fig. 3. The 1D-HMM for face recognition 
3. Feature values calculating of face image

The width of the face image is defined as $\mathrm{W}$ and height as $\mathrm{H}$. Face image is divided into some wrapped blocks. The height of each block is $\mathrm{L}$, and the wrapped depth is $\mathrm{P}$. the total number of the blocks is observing vector $\mathrm{T}$, and $\mathrm{T}=(\mathrm{H}-\mathrm{L}) /(\mathrm{L}-\mathrm{P}) \dashv 1$. The values of $\mathrm{L}$ and $\mathrm{P}$ affect the accuracy of recognition. With the increase of $\mathrm{P}$, the recogniton accuracy also increases. The small value of $L$ makes the recognition of the blocks ineffective. The increase of $\mathrm{L}$ makes cut feature probability increae. When the $\mathrm{P}$ is big, the value of $\mathrm{L}$ has no effect on system recognition. Reference [6] has a detaled discussion about the relation among P, L, and HMM.

4. Training of face HMM

An HMM is built for every face in the face images database, which is trained by 5 different face images of one person. The 2D-DCT transform coefficient vector can form the observing vector series by small-block-dividing method. The HMM parameters will be worked out by training with observing vector series $\mathrm{O}=\left\{\mathrm{o}_{1}, \mathrm{o}_{2}, \hbar, \mathrm{o}_{\mathrm{T}}\right\}$.

First, initialize the HMM $\lambda=\{A, B, \Pi\}$, and work out training data through dividing face image uniformly and from top to bottom. $\mathrm{N}$ is the state number of the model, $\mathrm{N}=6$. Observed vector serial concerning every state is used to calculate initial estimate of observed states probability matrix $B$. The initial values of $A$ and $\Pi$ are worked out of the face model's frame. Then, reestimate the model parameters by the method of maximal similar estimate arithmetic (Baum-Welch estimate arithmetic), and estimate convergence condition of $\mathrm{P}(\mathrm{O} \mid \lambda)$.

The model would be converged when the following formula (3) works. Training iterative process can be finished. Otherwise go on training. $\mathrm{C}$ is a given threshold beforehand.

$$
\left|\mathrm{P}\left(\mathrm{O} \mid \lambda^{(\mathrm{k}+1)}\right)-\mathrm{P}\left(\mathrm{O} \mid \lambda^{(\mathrm{k})}\right)\right|<\mathrm{C}(1)
$$

\section{Face recognition}

Observed vector serial is worked out from the face image for recognizing by the same method in train process, and its probability is calculated from the face image HMM. The formula is as follows:

$$
\mathrm{P}\left(\mathrm{O}^{\mathrm{T}} \mid \lambda^{(\mathrm{k})}\right)=\max _{\mathrm{n}} \mathrm{P}\left(\mathrm{O}^{\mathrm{T}} \mid \lambda^{(\mathrm{n})}\right)
$$

The face of No. $\mathrm{k}$ person corresponding to the face being recognized is identified from the database when formula (4) is sufficed.

The experiment is carried on the face images which are selected from the ORL face database as training unit. The system forms a training vector serial for each face image. The test recognition possibility is high.

The experiment proves this algorithm makes real time treatment feasible, for the algorithm is not easily affected by the changing of facial expressions, strongly resistant to noises, and robust. Further research is needed on another two problems concerning light and gesture. 


\section{Conclusion}

The face recognition system based on ARM and HMM has many advantages including small size, less calculating, high speed, and stable performance. So it can satisfy the needs for portable recognition devices. In the coming future, the embedded face recognition system will be widely used in security check-up, ID confirmation, entrance guard system, intelligent attendance check.

Acknowledgments. This research was supported in part by grants from 973 Program of Chinese Ministry of Science and Technology (2006CB303101), from the National Natural Science Foundation of China (60573059).

\section{References}

1. Kan, L., Qizheng, L.: Application of Image Gather Based on the S3C2410 Platform And Embedded Linux. CONTROL \& AUTOMATION 22(3-2), 125-127,168 (2006)

2. Gong, Y., Sakauchi, M.: Detection of Regions Matching Specified Chromatic Features. Computer Vision and Image Understanding 61(2), 263-269 (1995)

3. Yang, M.H., Kriegman, D.J., Ahuja, N.: Detecting Faces in Images: A Survey. IEEE Transactions on Pattern Analysis and Machine Intelligence 24(1), 34-58 (2002)

4. Nefian, A.V., Hayes, M.H.: Face detection and recognition using Hidden Markov Models. In: Proceedings of the International Conference on Image Processing, pp. 141-145 (1998)

5. Zhiliang, W., Yanling, Z.: An Expert System of Commodity Choose Applied with Artificial Psychology. In: IEEE International Conference on Systems, Man and Cybernetics, pp. 2326-2330 (2001)

6. Samaria, F., Halter, A.: Parameterization of stochastic model for human face identification. In: USA: Proceedings of the second IEEE workshop on application of computer vision (1994) 